\title{
COLLECTING RANDOMNESS FROM THE NET
}

\author{
B.Bencsath and I.Vajda \\ Dept. of Telecommunications, TU of Budapest
}

Key words: generation of random values, tests of randomness, good source of random data, private and authentic communication

\begin{abstract}
In this paper the problem of producing random binary blocks for security applications is considered. Random data is collected from network time delay measurements and its quality is checked by statistical tests. A special enhancement, a system of collector-servers is proposed and its security is analysed.
\end{abstract}

\section{INTRODUCTION: THE IDEA}

Good random values are very important ingredients for algorithms used in the field of data security. In the absence of sources that use physical randomness (such as electronic noise), random numbers of guaranteed quality are not readily available. Disregarding this aspect of security may prove dangerous, as these weakly random numbers may come from a much smaller space than expected, thus simplifying an attacker's task considerably.

This paper suggests a new method for producing high-quality randomness. It is based on the "butterfly effect", as applied to a large number of observed components. The Internet features a large number of network elements (routers, servers, etc.), and experiences a continuously fluctuating number of requests for different services. As a simple consequence, the time delay of an answer to any query from a client to a remote server will depend on a huge number of effects, i.e. on the instantaneous state of the net. Therefore time-delay measurements may 
plausibly be expected to carry useful randomness. The first part of the paper presents some typical results obtained from such measurements.

Even if we are able to generate randomness from measurements on the net, its use in security applications is not obvious at all. The reason behind this is an attacker's (theoretical) ability to silently track our measurements, e.g. the attacker is sniffing the client's LAN. Such an attacker might in principle be able to closely estimate the extracted random values. In the second part of the paper we propose a solution, a randomnesscollector-system (RCS), for this problem. The RCS is a set of servers residing on different host machines spread over the net, accepting requests from clients for collection of random binary data. The collected random data has to be safely transmitted to the client, hidden from the prying eyes of the attacker who would like to record the transmitted data. An appropriate protocol is suggested. We investigated also the problem of a nearby attacker who tries to estimate the collected data.

E-commerce security applications require random data to create good keys for cryptography, but many systems dont have a reliable random number generator. Special computers, like set-top-boxes, embedded web servers simply dont have a good source of data to feed a random number generator. Our goal was to provide a solution to these problems.

\section{1. THE RANDOM DATA}

\subsection{Measurement set up}

We have carried out extensive measurements on network time delay. Samples have been collected from two 'sources': the round trip time (RTT), measured by the standard ping'program and page download time from web servers. These remote points will be referred to as remote-servers (or target hosts) hereafter.

The ping "ICMP ECHO Request" resolves time in microseconds (it uses the default $C$ "struct timeval" structure, which contains a "long" number of microseconds). Therefore typical measurements could represent the response times with two bytes.

Our other randomness-collecting program was a simple script downloading a web page each second. A web page download is a much more complicated task than a simple Echo Request. Some webpages contain dynamic data that is created from a database. We expect the webpage download times to have more randomness than a simple ping, and making it 
harder to anticipate its value. The web page download time was also measured in microseconds by gettimeofdate() functions.

We have made extensive measurements on the response time of different remote-servers. (ping RTT and web page download times). Our programs saved the delay times to a database, enabling many different actions on the same data to be carried out.

\subsection{A simple statistical model}

A simple statistical model for the measured samples is the following: $x(t)=s(t)+n(t)$

where $x(t)$ is the observed sample, $s(t)$ is a slowly changing mean value and $n(t)$ a random fluctuation, and $t$ stands for the time parameter.

The mean value of RTT is typically around 2-10 milliseconds within a campus, and around 40-500 milliseconds for oversea connections. The RTT mean value depends on the Internet connectivity, on the length of the path between the two computers and on the time of day. The random component is determined by the net effect of many different factors. The net is huge and complex with continuously changing unpredictable load caused by end systems. In the end, a huge number of simultaneously changing effects determine a parameter of the net that is observed in measured samples. This can be modeled as a result obtained from a series of random variables. The first interesting question is the 'amount of randomness' in these variables.

In Table 1 . below we present a typical collection of measured values, where the type of the remote-server and elementary statistics of measured response time values (maximum, minimum, average, standard deviation) are shown.

Table 1 Elementary statistics of measured response time values (data in microseconds)

\begin{tabular}{|l|l|l|c|c|c|c|}
\hline $\begin{array}{l}\text { Server } \\
\text { No. }\end{array}$ & Type & $\begin{array}{l}\text { No. of } \\
\text { samples }\end{array}$ & Maximum & Minimum & Average & Std Dev. \\
\hline 1 & In-campus ping & 110291 & 13661 & 3167 & 4316 & 1725 \\
\hline 2 & Oversea ping & 199748 & 321779 & 173796 & 199432 & 20428 \\
\hline 3 & In-country ping & 429242 & 46696 & 3402 & 6503 & 5371 \\
\hline 4 & Continental ping & 427626 & 91281 & 8397 & 16550 & 13218 \\
\hline 5 & Continental ping & 430713 & 102995 & 64391 & 69600 & 4450 \\
\hline 6 & Local webpage & 417511 & 59000 & 1583 & 15603 & 7973 \\
\hline 7 & In-country webpage & 158078 & 435584 & 21132 & 191896 & 58441 \\
\hline 8 & $\begin{array}{l}\text { Continental } \\
\text { ping(sniffed) }\end{array}$ & 186716 & 46988 & 3535 & 7125 & 5409 \\
\hline 9 & $\begin{array}{l}\text { Continental ping } \\
\text { (sniffed) }\end{array}$ & 188671 & 41998 & 10717 & 14391 & 4060 \\
\hline
\end{tabular}


The average is significantly larger than one standard deviation in all measurements. This implies that the magnitude of the slowly changing component is - accordingly - larger than the typical range of random fluctuation. The standard deviation is well within $2^{16}$, suggesting that the randomness inherent in this set of population of samples will be well below $16 \mathrm{bits} / \mathrm{sample}$, and a conservative guess would place it at 8 bits/sample at most. In order to obtain a more accurate estimate we have processed the samples in different robust, plausible ways and checked the result by statistical tests.

\subsection{Processing of raw data}

Our aim was to improve the randomness properties of these measured values by applying simple transformations (some of them can be found in [1]). The following transformations were applied:

For a start, the extreme values were expurgated from the set of samples. Let the result be denoted by $x^{\prime}(t)$. We examined different plausible rules for further processing $x^{\prime}(t)$ :

Rule MA: The slowly changing component $s(t)$ has been estimated by the moving average (MA) computed over a sliding window of 20 samples. By subtracting this MA from the samples we obtain an estimate on the random component $n(t)$. Rule MA+L_8: First we subtract the moving average from the data then with the aim of improving randomness; then the low order byte (L_8) of this estimate is taken. Rule L_8: We simply take the low order byte of $\mathrm{x}^{\prime}(\mathrm{t})$. Rule LXORH: We make the XOR addition of the low order (positions $0-7$ ) and the second low order bytes (positions 8-15). Rule L_8 +par.: The 8 bits of the low order byte are added by XOR addition to produce 1 bit random information for every input byte. Rule L_8_U_2: The upper 2 bits of the low order byte are extracted. It is elaborated upon below.

\section{$1.4 \quad$ Statistical tests}

For assessing the quality of the obtained random values, we applied several statistical tests. The aim of these tests is to measure the deviation of the tested random data from the ideal coin tossing sequence. The following tests have been applied:

Maurer's test;Poker test;Monobit test;Runs test;Long runs test;Chi-square goodness-of-fit-test

With Maurers test [2] we used $L=8$ parameter due the low amount of available data. The expected value of the test for $\mathrm{L}=8$ with a perfect random 
number generator is $\mathrm{fTU}=7.183665$. Results from a good random source must not differ greatly from the expected value.

The Poker, Monobit, Runs and Longruns tests are part of the FIPS-140-1 and FIPS-140-2 standards. We used an implementation by Neal Bridges[3]. The monobit test counts the number of "l"s in a 20000-bit long bit stream. The poker test uses 20000 bits of data and tests 5000 contiguous 4-bit segments for probability. Due to the 20000-bit block length, these tests were run on more blocks of input values.

The runs test calculates the run lengths $(1 . .5$ and $6+)$, these "runs" have a sequence of consecutive bits of either all ones or all zeros. If the statistical result is within predefined intervals, the test passes. The long run test checks if a "run" with more than 26 bit exists in 20000 bits.

Our Chi-square goodness-of-fit-test implementation used cells defined by consecutive 8 bits ( 1 byte). The test doesnt succeed if the probability of the chi-square value is below about 1 percent. From $1 \%$ to $99 \%$ probability we cant say anything.

The Shannon entropy has also been calculated for 8 and 16 bits per sample. We have used some other tests too, such as [4], with similar results.

A few characteristic results are shown in Table 2.

Table 2: Results of statistical tests

\begin{tabular}{|l|l|l|l|l|l|l|l|l|}
\hline $\begin{array}{l}\text { Server } \\
\text { No. }\end{array}$ & $\begin{array}{l}\text { Type of } \\
\text { processing }\end{array}$ & $\begin{array}{l}\text { Maurer's } \\
\text { test }\end{array}$ & $\begin{array}{l}\text { Pok } \\
\text { er }\end{array}$ & $\begin{array}{l}\text { Mo- } \\
\text { nobit }\end{array}$ & Runs & $\begin{array}{l}\text { Long } \\
\text { Runs }\end{array}$ & $\begin{array}{l}\text { Chi Square } \\
\text { (Confidence } \\
\text { level \%) }\end{array}$ & $\begin{array}{l}\text { Shannon } \\
\text { Entropy (bit } \\
\text { size of sample) }\end{array}$ \\
\hline dev/mnd & L_8 & 7.184011 & Pass & Pass & Pass & Pass & $290.74(6.13)$ & $7.999448(8)$ \\
\hline dev/md & L_16 & N/A & & & & & N/A & $15.727350(16)$ \\
\hline 1 & L_16 & 5.479157 & Fail & Fail & Fail & Pass & $3406391(0)$ & $10.9458(16)$ \\
\hline 1 & MA & 5.524571 & Fail & Fail & Fail & Pass & $1834372.37(0)$ & $11.6761(16)$ \\
\hline 1 & L_8 & 7.168220 & Fail & Pass & Pass & Pass & $461.17(0)$ & $7.9969(8)$ \\
\hline 1 & MA+L_8 & 7.173395 & Pass & Pass & Pass & Pass & $316.51(0.52)$ & $7.9979(8)$ \\
\hline 1 & L_8+par. & 7.174118 & Pass & Pass & Pass & Pass & $244.15(32.25)$ & $7.9872(8)$ \\
\hline 5 & L_16 & 5.960379 & Fail & Fail & Fail & Pass & $3469926.25(0)$ & $12.5285(16)$ \\
\hline 5 & MA & 6.016704 & Fail & Fail & Fail & Pass & $3401189.50(0)$ & $12.6337(16)$ \\
\hline 5 & L_8 & 7.183341 & Pass & Pass & Pass & Pass & $259.87(40.37)$ & $7.9995(8)$ \\
\hline 5 & MA+L_8 & 7.183805 & Pass & Pass & Pass & Pass & $256.37(46.41)$ & $7.9995(8)$ \\
\hline 5 & L_8+par. & 7.183577 & Pass & Pass & Pass & Pass & $244.28(32.56)$ & $7.9967(8)$ \\
\hline 5 & L XOR H & 7.181106 & Pass & Pass & Pass & Pass & $286.22(8.71)$ & $7.9995(8)$ \\
\hline 8 & L XOR H & 7.186706 & Pass & Pass & Pass & Pass & $230.92(14.18)$ & $7.9991(8)$ \\
\hline 9 & L XOR H & 7.186481 & Pass & Pass & Pass & Pass & $261.22(38.10)$ & $7.9990(8)$ \\
\hline EGD_5 & L_8 & 7.185633 & Pass & Pass & Pass & Pass & $224.46(8.37)$ & $7.9995(8)$ \\
\hline 8 & L_8_U_2 & 7.180367 & Pass & Pass & Pass & Pass & $257.22(44.92)$ & $7.9960(8)$ \\
\hline
\end{tabular}


The first two rows of the table (/dev/rnd) contain data corresponding to the Linux standard /dev/random random number generator. It uses interrupt timing, block devices, keyboard and mouse movement to make good random numbers. The data is subject of entropy estimates and random refining tools (hash function). These rows are used for comparison purposes. The EGD_16 row is an output made by the "entropy gathering daemon' [5] program. We have modified the EGD program to use as a source of randomness $7.5 \mathrm{bits} /$ byte information (EGD parameter) from one of our previous tests (test no. 16 with lower 8 bits modifier). We checked the EGD's ability to produce as good statistics with our data feed as with other random sources.

Table 2 lends itself to several interesting observations:

The most important observation is that network latency times have enough extractable randomness. The raw data of delay times should be subjected to randomizing transformations. Relatively simple transformations can substantially improve the quality of the randomness. As it has been guessed above, 16 bits of raw data doesnt have 16 bits of usable random data.

Certain data we used is not long enough for a rigorous analysis. For chisquare and other tests we need a number of cells with data. If we have 256 different cells, we need at least 2560 bytes of data to make a minimal test. If we use 65536 different cells, then many more samples are needed. Some random number generator tests (like diehard) need megabytes of random data. If we dont use infinite random numbers for statistical tests, there is a chance of a bad statistical decision. The amount of data we used in the tests is limited to the speed of the data collection. It is clear, however, that the delay times do have a certain amount of extractable random data.

\section{THE RANDOMNESS-COLLECTOR-SYSTEM}

\subsection{Default configuration: distributed collection}

The system (Fig.1) consists of a set of collector-servers (RC), which means $\mathrm{N}$ servers residing on different host machines spread over the net. This RCS accepts requests from clients to collect random binary data. A "Target Host" is the target of the delay measures (RTT). These measures are made by the randomness collectors (RCs). 


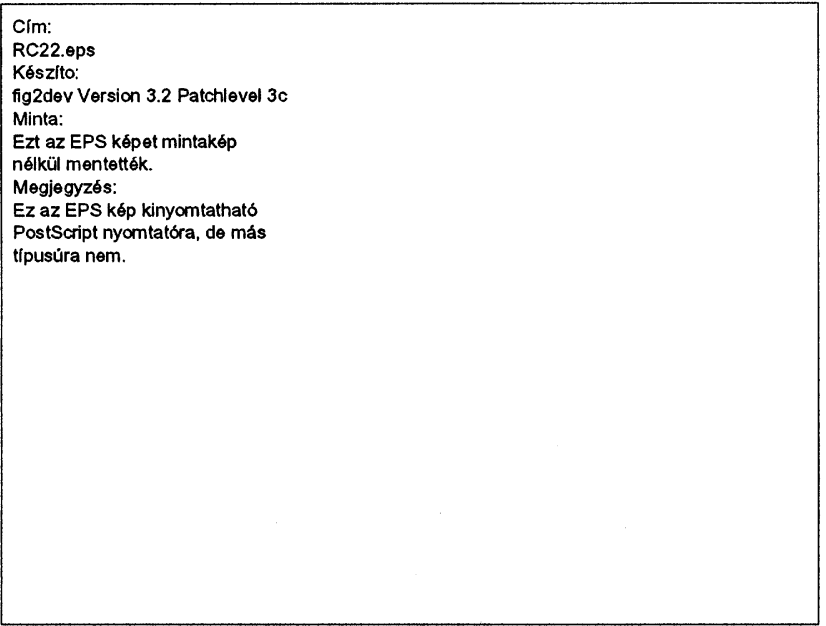

Figure 1 The randomness-collector-system

The communication path between clients and collector-servers must provide confidentiality and authenticity. This means that data transmitted from the collector-server to the clients should only be readable by the client, and the client should also be able to check the authentic origin of the received data.

One possible implementation of these security requirements can be based on public key technology. All collector-servers and clients are assigned appropriate key pairs for performing public key encryption and decryption operations. (This implies that an initial randomness' exists in the system) As usual, confidentiality can be ensured by public key encryption. Authenticity can be ensured by performing the decryption operation using secret keys. Available cryptographic primitives permit different solutions to be designed [7]. For example the following two-step protocol meets the above security requirements:

$\mathrm{C} \rightarrow \mathrm{S}:$ id, $\mathrm{k}_{\mathrm{p}, \mathrm{C}}, \operatorname{Cert}\left(\mathrm{k}_{\mathrm{p}, \mathrm{C}}\right)$

$\mathrm{S} \rightarrow \mathrm{C}:\{\mathrm{M}\} \mathrm{k}_{\mathrm{p}, \mathrm{C}},\left\{\mathrm{M}^{\prime}, \mathrm{id}\right\} \mathrm{k}_{\mathrm{s}, \mathrm{S}}$

where

id: session identifier

$\mathrm{k}_{\mathrm{p}, \mathrm{C}}$ : public key of C

$\mathrm{k}_{\mathrm{s}, \mathrm{s}}$ : secret of $\mathrm{S}$

Cert $\left(\mathrm{k}_{\mathrm{p}, \mathrm{c}}\right)$ : certificate for public key

\{\} $\mathrm{k}$ : encryption in key $\mathrm{k}$

M: data to send

$M^{\prime}$ : fingerprint of data

A hybrid key solution could be based on Diffie-Hellman keys.

It is required that the hosts of collector-servers and clients are secure in the sense their HW/SW subsystem performing these security operations 
cannot be compromised. In any centralized security system the centers are sensitive parts.

After the client received $N^{\prime}$ random blocks, $R C$ _i_rand, $i=1, \ldots, N^{\prime}, N^{\prime} \leq N$, it combines them into one block. By default it calculates the XOR of all $R=X O R_{i=1, N^{\prime}}\left(R C_{-} i_{-}\right.$rand $)$ input blocks.

\subsection{Access via proxies: centralization}

Clients send their request to a single network element, the proxy. (sample on Fig.2) This proxy collects and mixes the input data that has been collected by the RCs. After obtaining random data, a connection is built with clients and the data is transmitted for further use. Such a proxy can help resource-constrained clients to decrease the computational power and network bandwidth needed for secure communications.

\section{SECURITY ANALYSIS}

\subsection{Estimation of measured samples by an attacker}

How accurately a physically nearby attacker could estimate the collected random samples is a vital question. As a worst case, assume that the attacker knows the actual remote connections (and also the time order of corresponding samples) and tries to replicate the procedure done by the client. We have made the following measurement regarding such an attacker:

We used one computer to collect data (pinging a host). Another computer on the same hub has used a packet sniffing software to detect the data sent by the original host. Let $\mathrm{X}$ and $\mathrm{Y}$ denote the associated random samples measured by the client and the attacker respectively. We have calculated the conditional Shannon entropy $\mathrm{H}(\mathrm{X} \mid \mathrm{Y})$, which measures the ambiguity about the actual value of $\mathrm{X}$ for the attacker, when $\mathrm{Y}$ is known to him. Consider the following model:

$$
\begin{aligned}
& x(t)=h(t)+r_{c}(t) \\
& a(t)=h(t)+r_{a}(t)
\end{aligned}
$$

where $x(t)$ is observed by the client, $a(t)$ is observed by the attacker, $h(t)$ is the value at the hub, components $r_{c}(t)$ and $r_{a}(t)$ are produced by the 
HW/SW complex of the client's host and the attacker's host, respectively. From (3) it follows that

$$
x(t)=a(t)+\left(r_{c}(t)-r_{a}(t)\right)
$$

The difference between data collected by the client and the attacker is the difference of time delays caused by the hosts. Therefore we calculated the conditional entropy

$$
H\left(a(t)+r_{c}(t)-r_{a}(t) \mid a(t)\right)=H\left(r_{c}(t)-r_{a}(t)\right)
$$

where the equality is obtained from the independence of effects generating variable $a(t)$ and variables $r_{c}(t)$ and $r_{a}(t)$. It is a plausible assumption that variables $r_{c}(t)$ and $r_{a}(t)$ are independent and identically distributed random variables, therefore the conditional entropy is between values $H\left(r_{c}(t)\right)\left(=H\left(r_{a}(t)\right)\right)$ and $2 H\left(r_{c}(t)\right)$. The value of conditional entropy is generated by two sources, while the value of variable $x(t)$ is affected only by one of them.

We have measured $\mathrm{H}(\mathrm{X} \mid \mathrm{Y})=5.3$ (delay times to server no. 8). This means that even a nearby attacker, who is able to track the collecting activity of the client, experiences a fairly high ambiguity about the random values seen by the client.

The test with the L_8_U_2 modifier uses just the upper 2 bits of the low order byte. We expect that it doesnt contain randomness from the hardware $\left(r_{c}(t)\right.$ and $\left.r_{a}(t)\right)$, only randomness from the network.

\subsection{Protecting RCs from attacks}

Protecting RCS from sniffing It is assumed that an attacker is technically able to silently track our measurements launched from a given (known) host on the net. The RCs are collecting random data from different Internet connections. If anyone can sniff the Internet connectivity of an RC, the information in the time delay might compromised. We can conservatively assume that such kind of attack against a machine hosting a collector-server cannot be prevented.

However such an attacker must be physically (propagation time) close to the measuring machine. Therefore it is a plausible assumption that this capability of the attacker should exponentially decrease with the number of machines from which the measurement is simultaneously carried out. Consider the following simple statistical model. Assume that an attacker is able to track measurements from RCS independently and the probability that he can track a given machine is $p, 0<p<1$. Consequently

$P($ an attacker can track $j$ machines simultaneously $)=B(p, N, j)$,

where $j=1,2, \ldots \mathrm{N}$ and $\mathrm{B}$ is the binomial distribution with parameters $\mathrm{p}$ and N.. Here $p$ is typically a low value, say $p<1 / 30$ could be considered a very conservative estimate (calculations based on [6]). The probability 
$\mathrm{P}^{*}=\mathrm{p}^{\mathrm{N}}$ that the attacker is able to simultaneously attack all machines of RCS decreases exponentially with the value of parameter N (size of the RCS).

Hiding association between measured samples and clients The servers in the system collect random data for lots of simultaneous requests. Because the servers own secret keys, they can distribute the random samples among requesters. If the system is centralized, then the center could perform this task of distribution.

It is easy to see that enhancing the system with such a properly working (i.e. preloaded with lots of requests) association hiding option substantially decreases the risk of attackers sniffing around the inputs of collectors.

Attacking communication path between clients and servers When an attacker is interested in a specific client, it is plausible that instead of attacking lots of collectors spread over the net, he would try to get close to the client with the aim of observation and/or manipulation of random data arriving from the collector system. The data should be protected against the following attacks: eavesdropping, fabrication of bogus message and replay of old compromised messages. The needed cryptographic technology is available and well elaborated [7]. We can protect the data against eavesdropping by applying encryption. (Note that the used encryption technique can be unique because after decrypting the message we dont have to get the same random data as the original.) Using secret keys can prevent fabrication of a message. Insertion of nonce values (e.g. session identifier) into the messages can prevent the third type of attack.

Compromising the hosts of servers and clients It is assumed that the servers and clients can protect their secret keys from an attacker. The whole RCS needs a proper initialization. If a part of the subnet is compromised, then the compromised keys should be changed and the system should be verified against backdoors as in the case of other Internet servers.

DoS attack against the servers It is well known that the Internet is unfortunately highly vulnerable to DoS attacks. Our distributed collector system shows some structurally inherent robustness against DoS attacks. A centralized configuration is much more vulnerable.

The amount of randomness Network delay is going to be smaller and smaller. If we have very fast Internet connections, there is less randomness in network time delays. In fact, network delays wont go under a minimum, which is the speed of light. An Internet connection around the planet can be $20.000 \mathrm{~km}$, which needs about 66 milliseconds. On the other hand, as the network links get faster, lines carry ever-increasing numbers of connections that add more randomness in network time delay variation. QoS may be a concern, as it enforces rules for network time delay variation, but not with infinite precision (and QoS is often only available on small subnetworks, not in general use). 
Different platforms use different hardware and different hardware clocks, which have an effect on the collected data. On many systems, the clock does not resolve time to microseconds, etc. We are not saying that every operating system and every computer is fit to be an RC. We need RCs for clients that are unable to make good random numbers for themselves, so we can prescribe even microsecond capability on the $\mathrm{RC}$ hardware. To eliminate problems in the lower bits of the time delay, we use the bit mixing functions as described.

\subsection{Limited usage of randomness}

The random data is protected by some kind of cipher algorithm from the randomness collector to the client host. If the client gets $\mathrm{N}$ random bits, but the channel is protected with a cipher with a key size of $M$, then an exhausting attack for an application that uses the $\mathrm{N}$ random bits should run with a maximum of $2^{\min (\mathrm{N}, \mathrm{M})}$ trials, assuming the presence of an eavesdropper on the network.

For this reason, the random data generated by the random network should not be used to make larger keys than the strength of the cipher that protects the submitted random data. Using mixing functions with initialization keys or combining the system with various other random source and other solutions could help to lower the chance of the random data getting compromised. With some modifications on the model (special use of random data source, selecting-mixing, etc.) we can enhance the effect of the encryption. With such modifications an attacker would have to try much more possibilities than $2^{\min (\mathrm{N}, \mathrm{M})}$.

\section{REFERENCES}

[1] Hoel,P.G. - Jessen, R.J, Basic Statistics for Business and Economics. John Wiley \& Sons Inc., 1982

[2] Maurer, Ueli M., A universal statistical test for random number generators, Journal of Cryptology, vol.5, no. 2., 1992, pp. 89-105.

[3] Security Requirements for Cryptographic Modules, Federal Information Processing Standards Publication 140-1, 1994, http://csrc.ncsl.nist.gov/fips, Implementation: Neal Bridges, 2000.

[4] Walker,John, Ent, A Pseudorandom Number Sequence Test Program, 1998, http://www.fourmilab.ch/

[5] Warner,Brian, EGD: an Entropy Gathering Daemon v0.8,

[6] Dr. Howard,John D., An analysis of Security Incidents on the Internet 1989-1995, 1997 http://www.cert.org/research/JHThesis/Chapter9.html

[7] Schneier,B.,Applied Cryptography, Part I, Cryptographic Protocols, John Wiley \& Sons Inc.,1996. 